\title{
Von Kopf bis Fuß - Pflanzen mit Namen menschlicher Körperteile
}

Hilke Steinecke

Wenn der Palmengarten abends seine Tore schließt, können dennoch während spezieller Führungen in den Abendstunden Pflanzen erkundet werden. Es besteht für Gruppen die Möglichkeit, über die Grüne Schule nach Feierabend Führungen zu bestimmten Themen zu buchen. Zudem gibt es ein festes Palmengarten-Angebot an Führungen und Vorträgen, an denen auch einzelne Personen teilnehmen können.

Besonders an kalten Winterabenden ist der Kontrast zwischen den eher ungemütlichen Temperaturen draußen und der tropisch-warmen Atmosphäre in den Schauhäusern des Tropicariums reizvoll. Von Januar bis März 2007 standen wöchentlich etwa zwei Stunden dauernde Führungen zu Pflanzen, deren Namen etwas mit menschlichen Körperteilen zu tun haben, auf dem Programm. Ulrike Brunken, Heidrun Janka, Peter Schubert und Hilke Steinecke führten jeweils 40 Personen, aufgeteilt in zwei Gruppen, von Kopf bis Fuß durch die Gewächshäuser. Jedem Haus war dabei eine andere Körperregion gewidmet und wir versprachen, dass alles, worüber wir berichteten, Hand und Fuß hat.

Zur Begrüßung versammelte sich die Gruppe im Eingangsbereich vor der Weltkugel. Überall auf der Welt gibt es bestimmte Pflanzen, die in der Volksmedizin bei Krankheiten bestimmter Organe zum Heilen eingesetzt werden. Gemäß der Signaturlehre des Paracelsus haben manche Pflanzen eine bestimmte Signatur und lassen damit angeblich erkennen, gegen welche Krankheiten sie helfen. Als Beispiele wurden Lungenkraut, Leberblümchen, Milzkraut und Augentrost gezeigt. Manchmal wurden auch Pflanzenorgane mit ganzen Lebewesen verglichen wie z. B. die menschenähnlich verzweigten Wurzeln von Alraune oder Ginseng. Und es gibt

Abb.1 (oben): Kopfnüsse als Erinnerung an die Führung. Abb. 2 (unten): Korbblütler haben Köpfchen. einige Namen, die sowohl Menschen als auch Pflanzen tragen. Wir freuten uns, wenn sich in der Gruppe eine Iris, Viola, Erika, Rosa, Angelica, Schwarzäugige Susanne oder ein Fleißiges Lieschen, Flammendes Käthchen sowie ein Guter Heinrich befanden.

Die Kopf-Station befand sich in der Halbwüste. Einen Bezug zum Haar ließen dort Venushaar-Farn und Greisenhaupt-Kakteen erkennen. Das Bubiköpfchen zeigte seine kurz geschnittene Blattfrisur. Der durch zerfaserte
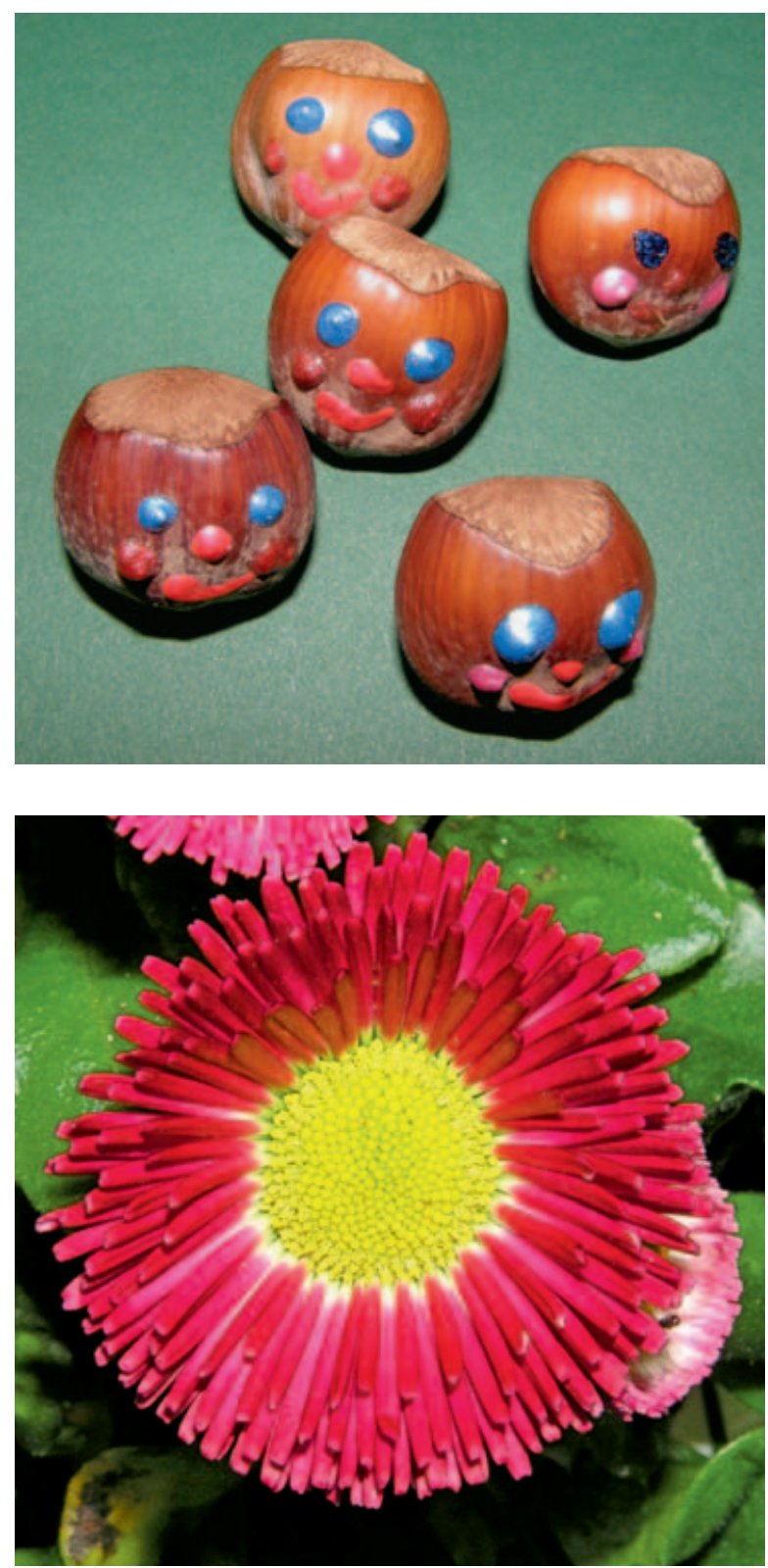

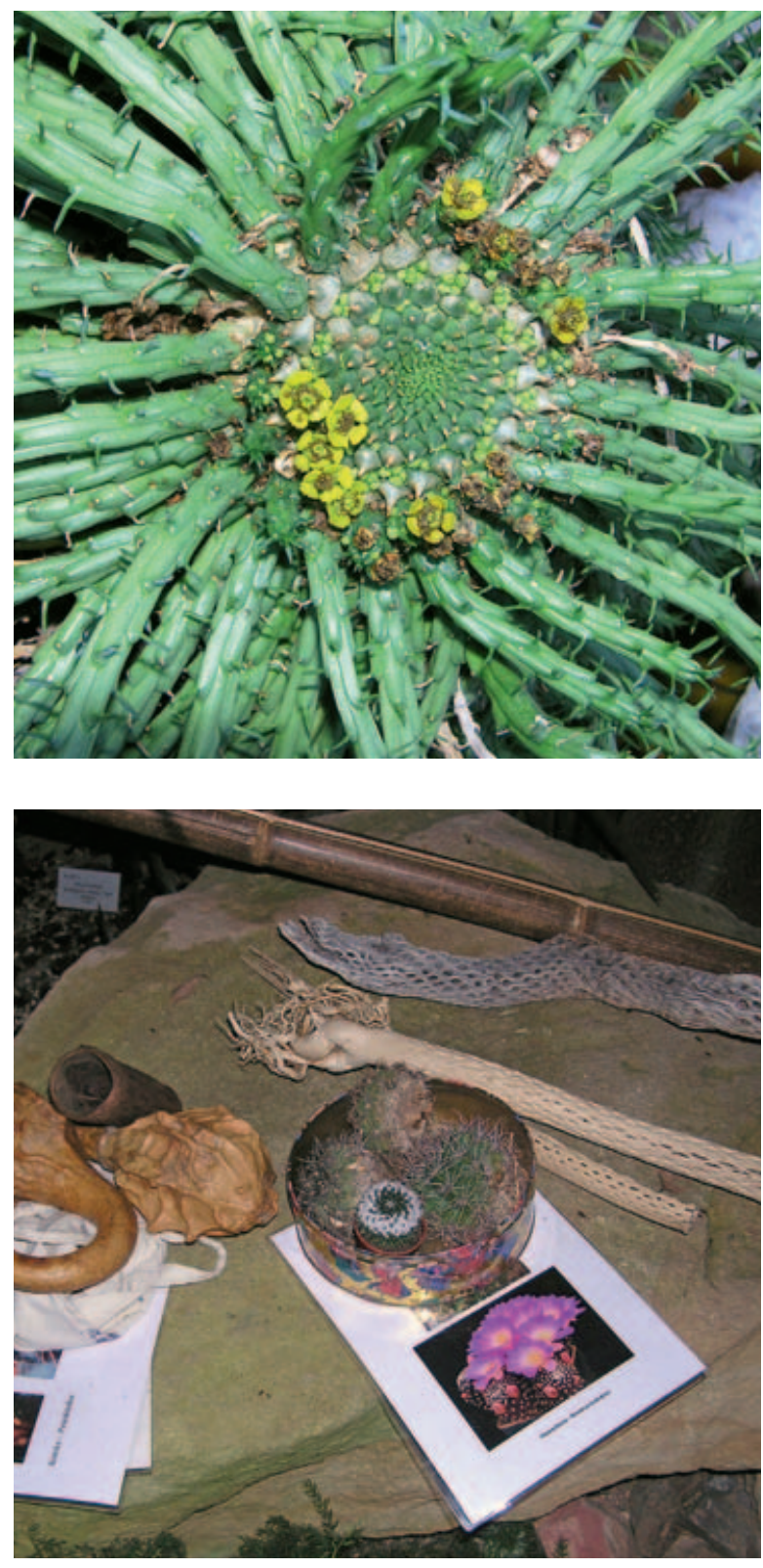

Blattscheiden wollig behaarte Stamm einer Coccothrinax crinata-Palme (old man palm) erinnert ihrem Volksnamen nach wohl mehr an Bart-als Kopfhaar. In der Nebelwüste wurde ein Medusenhaupt (Euphorbia caput-medusae) gezeigt. Die sukkulenten Triebe dieses Wolfsmilchgewächses erinnern an Schlangen, die nach griechischer Mythologie der Medusa aus dem Haupt wachsen. Die Geschichte ihrer Enthauptung war traurig, bald kullerten Hiobstränen. Dies sind die porzellanartigen Früchte eines mit dem Mais verwandten Grases (Coix lacrima-jobi), die in tropischen Ländern zu Ketten aufgefädelt werden. Erwähnenswert waren auch ganze Pflanzenfamilien wie Lippen- und Rachenblütler. Zur Erinnerung an die Kopf-Station bekam jeder eine „Kopfnuss“, eine mit einem Gesicht bemalte Haselnuss. Kopfnüsse werden auch in Südamerika auf Märkten den Touristen angeboten, dann sind es meist Kokosnüsse, in die ein Gesicht hineingeschnitzt wurde.

Im Savannenhaus beschäftigten wir uns mit dem Rumpf. Hier gab es Spannendes über Brustwarzenkakteen (Mammilaria), Busenragwurz (Ophrys mammosa) und Leberwurstbaum (Kigelia africana) zu erfahren. Extrakte aus den Früchten des Leberwurstbaumes stärken das Bindegewebe und werden zur Straffung von Haut und Busen verwendet. Den ganzen Körper stützt das Gerippe. Ein Beispiel einer „Rippennamen-Pflanze" ist der Rippenfarn (Blechnum spicant) mit seinen rippenförmigen fertilen Wedeln. Unter unseren Utensilien hatten wir auch das Gerippe eines Kaktus, einen frei gewitterten Echinocereus-Zentralzylinder. An anderer Stelle gab es als Ergänzung Blattadern, Blut der Drachenblut-Palme (Daemonorops draco) und die Samen der Steinnuss-Palme (Phytelephas macrocarpa), die knochenähnliche Konsistenz haben und zum Schnitzen verwendet werden. Der Speckbaum (Portulacaria afra) mit seinen dickfleischigen, sukkulenten Blättern ermahnte uns daran, der Fettleibigkeit entgegenzuwirken. In Abhängigkeit von den bevorzugten Stellen, an denen sich die unbeliebten Speckpölsterchen bilden, unterscheidet man zwischen Birnenund Apfeltyp. Ersterer kommt häufiger bei Frauen, letzterer bei den so genannten Bierbäuchen einiger Männer vor.

In den Häusern der feuchten Tropen widmeten wir uns den Gliedmaßen. Wir berichteten über Bananenhände, gefingerte Blätter und Buddhas Hand. Letztere ist eine Citrusfrucht (Citrus media'Sarcodactylus') mit fingerförmigen Auswüchsen. Die duftende Frucht symbolisiert die Gebetshaltung BuDDHAs und wird in China auf Hausaltäre gelegt. Eine gefriergetrocknete Frucht erzielte große Aufmerksam-

Abb. 3 (oben): Medusenhaupt in Blüte.

Abb. 4 (unten): An der „Rippen- und Brust-Station“. 
keit. Nach einer humorvollen technischen Anleitung zum Öffnen einer Banane durfte ein Teilnehmender die krumme gelbe Tropenfrucht verzehren.

„... Banane mit der Haltehand um die untere Hälfte fassen (Linkshänder mit der rechten), Fruchtstiel (vorstehende Verlängerung am oberen Ende) muss nach oben gerichtet sein. Der Druck der Haltehand ist so einzustellen, dass die Banane nicht gequetscht wird, andererseits aber sicheren Halt für den bestimmungsgemäßen Verbrauch gewährleistet. Die Bedienungshand darf nun den Fruchtstiel zwischen Daumen und Zeigefinger nehmen, diesen leicht abbiegen und mit einem festen, kontinuierlichen Ruck nach unten die Verpackung auftrennen. Verbleibende Verpackungsstreifen werden in gleicher Weise abgezogen ..."

Wir nahmen den Fachausdruck „genetischer Fingerabdruck" wörtlich und schenkten jedem ein Buchzeichen, verziert mit unseren eigenen Fingerabdrücken, die wir zu lustigen kleinen Vögeln ergänzt hatten. In der Mangrove stand am Wegesrand eine Schraubenpalme (Pandanus) mit einer sprossbürtigen Wurzel, die mit ihren fünf Verzweigungen wie ein Fuß wirkte. Viele Geschichten gab es auch um Knoblauchzehen und die so beliebte südafrikanische Heilpflanze Teufelskralle (Harpagophytum procumbens). Die Krallen der Frucht bleiben nicht nur leicht im Fell oder an den Hufen von Tieren hängen. Die Widerhaken verankern sich auch leicht in menschlicher Haut. Einen Zungenbrecher bot die Geschichte von Franz FüHmann über das Drehzehweh der Schneeseekleerehfee:

„... Guten Tag, beste Heckenhexe mit den Hackenhaxen! Hast du etwas gegen mein schreckliches Drehzehweh? Aber klar, sagte die Hexe, ich werde dir nun einen Schneeseekleerehfeedrehzehwehtee brauen. Die Heckenhexe mit den Hackenhaxen nahm Blätter von sieben Kräutern und kochte daraus in einem Kessel

Abb. 5 (oben): Öffnen einer Banane nach technischer Gebrauchsanweisung.

Abb. 6 (unten): Frauenschuh als „Bekleidungsempfehlung“ für den Heimweg.
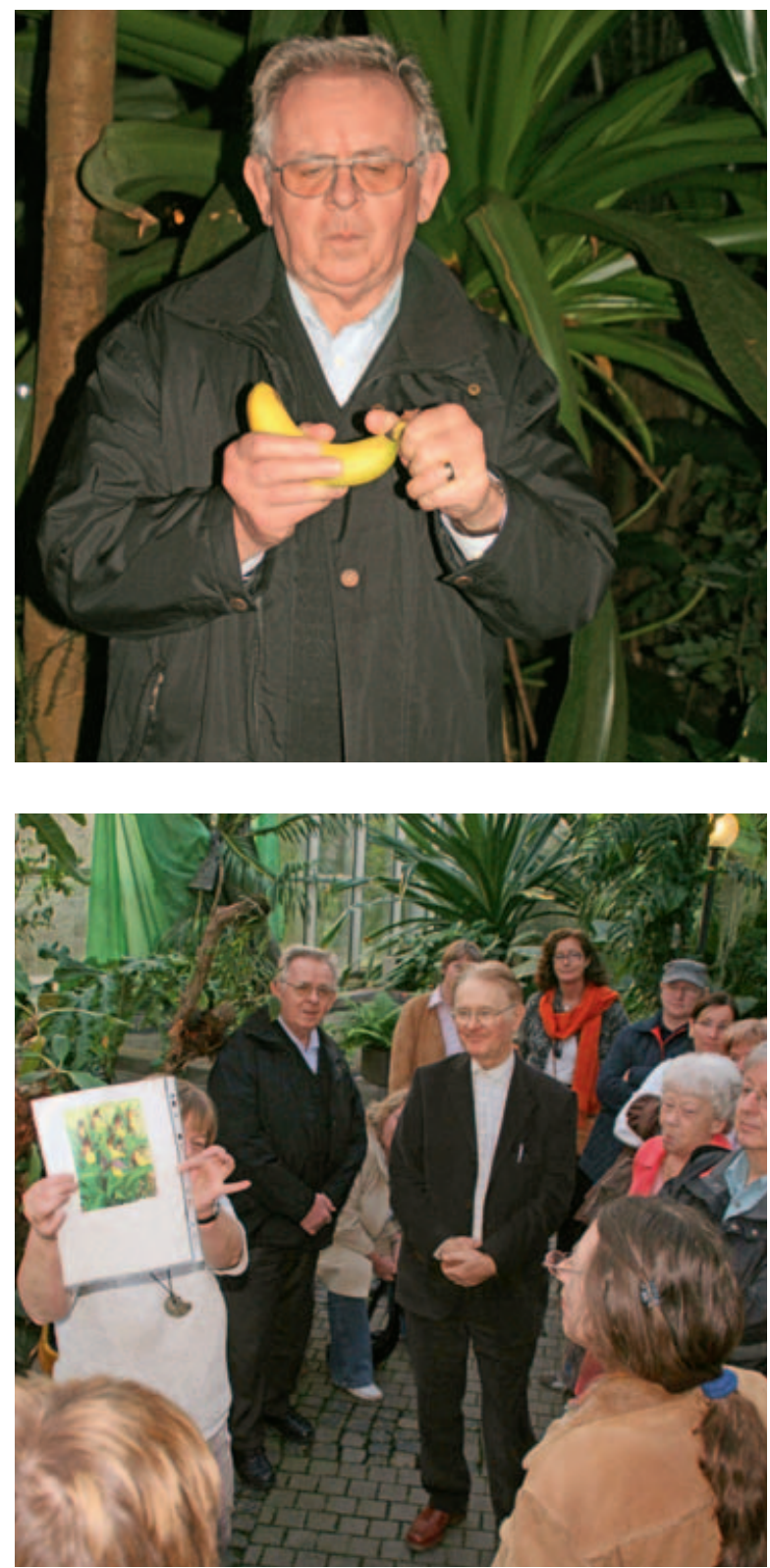

einen hellgelben Tee. Trink das, nette Schneeseekleerehfee! Da trank die Schneeseekleerehfee auf einen Zug den Schneeseekleerehfeedrehzehwehteekessel aus, und als der Schneeseekleerehfeedrehzehwehteekessel ausgetrunken war, hatte der hellgelbe Schneeseekleerehfeedrehzehwehtee das Schneeseekleerehfeedrehzehweh aus dem Schneeseekleerehfeedrehzeh der Schneeseekleerehfee weggehext, und da stieß die glückliche Fee ein lautes Juchhe aus ..."

Weitere Stationen der Führung waren Orchideen, die Hodenblumengewächse, Avocado (was von der aztekischen Bezeichnung für die Frucht $=$ Hodensack abzuleiten ist), Amorphophallus, Clitoria und ein Feigenbaum. Die 
rundlichen, vielkernigen Feigenfrüchte wurden mit einem Herz verglichen, galten als Symbol für Sexualität, Liebe und Fruchtbarkeit. Vom Bau der Feige konnte sich jeder anhand einer süßen getrockneten Wildfeige überzeugen.

Zum Abschluss trugen wir ein Gedicht über die Liebe im Garten vor.

\section{Liebe im Garten}

Ein Kohlkopf stand neben einem Rosenstock und schaute verliebt wie ein Ziegenbock tagtäglich der Rose bleichende Pracht. Und eines Tages - das heißt bei der Nacht, da hielt der Kohlkopf es nicht mehr aus, er musste aus seinem Kohlherz heraus! Er kniete nieder, er seufzte, er schwur: „Ich liebe dich Rose - dich liebe ich nur!“ und hat, wie das so zu geschehen pflegt, der Rose sein Kohlherz zu Füßen gelegt.

Und die Rose???????

Im Dunkeln hat's niemand gesehen was zwischen den beiden ist weiter geschehen! Im nächsten Jahr - was war das wohl? stand neben der Rose - Rosenkohl.

Für den Heimweg durch Wind und Kälte gaben wir die Empfehlung mit, Frauenschuh, Frauenmantel und Sturmhut aufzusetzen, alle Mitbringsel in das Hirtentäschel zu stecken und im gemütlichen Heim Sockenblume, Pantoffelblümchen und Schlafmützenmohn hervorzuholen.

\section{Gärtnerisch-botanische Literatur}

\section{Helga Dietrich}

\section{Wo liegt das Abenteuerland? - Mit Rätseln, Reimen, Rezepten und Witzen}

EchinoMedia Verlag, Bürgel, 48 Seiten, zahlreiche farbige Zeichnungen und Schwarz-weiß-Bilder zum Ausmalen von Kerstin Ramm, 9,90 Euro; ISBN 978-3-937107-13-4

In diesem Buch erzählt die kleine LAUra von ihren Abenteuern, die sie zusammen mit ihrer Großmutter erlebt. Dabei durchlaufen sie die vier Jahreszeiten und entdecken immer wieder neue Dinge, die für die entsprechenden Jahreszeiten typisch sind. LAURA ist immer auf der Suche nach dem Abenteuerland, wobei sie doch eigentlich schon genau drin steckt. Die Botanikerin Helga Dietrich hat sich zu diesen Geschichten von ihrer eigenen Enkeltochter, der sie die Natur erklärt, inspirieren lassen.
Es ist ein gelungenes Kinderbuch mit einer spannenden Geschichte zum Vorlesen und vielen bunten Bildern zum Anschauen. Es werden mit dem Durchlaufen der einzelnen Jahreszeiten deren wichtigste Merkmale anhand von Bildern verdeutlicht. Dabei werden auch Pflanzen und Schmetterlinge abgebildet und erklärt. Zudem bringt das Buch viel Spaß beim Lösen von Rätseln. Anzumerken sei dabei allerdings, dass sich der Text der Geschichte eher auf Vorschulkinder bezieht (Laura wird bald 5 Jahre alt), die Rätsel (z. B. Kreuzwortsrätsel) erst gelöst werden können, wenn man lesen und schreiben kann. Aber sicherlich bereiten auch den Kleineren die Bilder zum Ausmalen, die Witze und Reime viel Freude. Ebenfalls sind leckere, etwas augefallene Rezepte mit Gewächsen von der Wiese zum Ausprobieren dabei. Wer bekommt da nicht Lust auf einen Löwenzahn-Sirup?

AnN-Kathrin Schollek 